\title{
The Determination and Practical Application the Kinetic Constants of Destruction of Rocks with Modified Zhurkov's Formula
}

\author{
Dmitry Sirota $^{1 *}$, Vadim Ivanov ${ }^{1}$ \\ ${ }^{1}$ T.F. Gorbachev Kuzbass State Technical University, 650000, 28 Vesennyaya St., Kemerovo, Russia
}

\begin{abstract}
Any kind of destruction is bound to microckracks. One of the theoretical model of microcracking is kinetic concept of strength (KCS) by S.N. Zhurkov. This theory allows to associate the time to failure with the activation energy and activation volume in single formula. But however, there are options for destruction in which this formula is incorrect. In this article we presents new modification of Zhurkov's formula. Also, we present two possible practice applications of kinetic constants of the destruction.
\end{abstract}

\section{Introduction}

The problem of durability is impotent for all types of mining. Early deterioration and destruction of used structures can lead to technogenic accidents and human casualties.

One of such technical facilities is automobile roads of mining enterprises, the quality of which is very important for the technical and economic efficiency of the enterprise. The roads quality of the enterprises for extraction of mineral is in the open way especially important. It is caused by difficult climatic conditions: rainfall, temperature drops, the long winters, mechanical influences.

In the underground mining and seismology the very impotent problems are the forecasting the energy, location of the rock burst source and the duration of the source forming. These tasks are related to solving a direct problem about an electric field of the rock burst source or technogenic earthquake in the atmosphere.

One of methods by calculation of roads durability or the duration of the source forming is based on S.N. Zhurkov's formula [1]. This formula has been received as generalization of experimental data on destruction of laboratory samples at their stretching. However real loadings can be both on compression and on stretching; and, in case of a paving, loads of compression are main. Therefore calculation of durability and kinetic constants of durability on the basis of Zhurkov's formula isn't reasonable. Currently, there are several practical applications of KCS and modifications of the basic Zhurkov's formula [2 - 15].

Let us in the following article 1) present a new modification of S.N. Zhurkov's formula, which is based on experimental data on the destruction of samples (composite and rocks)

\footnotetext{
${ }^{*}$ Corresponding author: sdy.pm@kuzstu.ru
} 
under compression; 2) to present its application to the calculation of the durability of the road surface in the conditions of a frost.

\section{Result and discussion.}

\subsection{The existing formulas and their modifications.}

The S.N. Zhurkov et all. KCS divides deformation process of materials into two stages: the chaotic uncorrelated creation of microcracks and creation the main crack of a gap.

The transition from the first stage to the second one is comes when the breaking criterion is executed which is presented by a formula [1]

$$
n^{-1 / 3} * l^{-1} \approx e
$$

where $n$ - average microcracks concentration, $\mathrm{m}^{-3} ; l$ - average microcracks linear size, $\mathrm{M} ; e$ $\approx 2,72$ - average distance between cracks in an unit volume exemplar in shares of their average size $l$.

The S.N. Zhurkov et all. [1] showed that the durability of the first stage can be calculated as

$$
\tau=\tau_{0} * \exp (\beta) * \exp (-\alpha \cdot \sigma)
$$

where $\alpha=\Omega *(k T)^{-1}, \beta=U_{0} *(k T)^{-1}, \tau_{0} \approx 10^{-13}$ - a typical period of atomic fluctuations, $\mathrm{s} ; \Omega$ - the stress sensitivity factor, $\mathrm{m}^{3} ; U_{0}-$ the zero stress activation energy, $\mathrm{J} ; k-$ Boltzman constant, $\mathrm{J} /{ }^{0} \mathrm{~K} ; T$ - the absolute temperature, ${ }^{0} \mathrm{~K} ; \sigma-$ the average external stress, $\mathrm{Pa}$.

The further research showed that the formula (2) is not universal. For example, more suitable for elastomers the time-to-fracture can be calculated as [9]

$$
\tau=\tau_{0} * \exp (\beta) *\left(\frac{\sigma}{E}\right)^{-\alpha \bullet E},
$$

where $E-$ is Young elastic modulus, Pa.

For polymers and annealed metals, the dependence of durability on load can be calculated as [10]

$$
\tau=\tau_{0} * \exp (\beta) *\left(\frac{\sigma}{\sigma_{P}}\right)^{-n},
$$

where $\sigma_{P}$-dimensional adjustable value which has not a physical meaning, $\mathrm{Pa}$; the value $n \sim n\left(U_{0}, k, T\right)$.

We can see that

1) All three formulas $(2,3,4)$ contain dependences from activation energy which means using the Gibbs distribution and differ in the way to evaluate the effect of stress changes on the sample;

2) Dependences $(3,4)$ have a similar form, but they differ in principle in the sense of physical interpretation the contained parameters.

Let us determine the relationship between time $\tau$ and some parameter that can be registered and measured. Since the largest defects (microcracks) emit electromagnetic pulses (EMP), the number of pulses that can be registered can be considered as the value of $N$. 
In [3], the authors formulated a kinetic model for the accumulation of microcracks, including the rate of crack formation, the Bailey destruction irreversibility condition, and the concentration fracture criterion (1)

$$
N^{\prime}(t)=N_{\max } / \tau, \int_{0}^{\tau} N^{\prime}(t) d t=N_{\max }
$$

where $N_{\max }$ - the maximum number of cracks that accumulates in the sample at the time of its destruction and allows us to describe the accumulation of structural damage when the effective stresses and temperatures are arbitrarily dependent on the time.

To determine the dependence of the number of pulses $N$ on the stress value, we integrate (5) under the condition of a linear increase in the stress $\sigma=\sigma^{\prime} \cdot t: N(\sigma)=\int_{0}^{t} \frac{N_{\max }}{\tau} d t$, hence in the case of the dependence (2) we obtain the expression

$$
N^{T}(\sigma)=\frac{N_{\max }}{\tau_{0} \cdot \sigma^{\prime}} * \exp (-\beta) * \frac{\exp (\alpha \cdot \sigma)-1}{\alpha},
$$

and in the case of the dependence (3) we obtain expression

$$
N^{T}(\sigma)=\frac{N_{\max } \cdot E}{\tau_{0} \cdot \sigma^{\prime}} * \exp (-\beta) * \frac{(\sigma / E)^{\alpha \cdot E+1}}{\alpha \cdot E+1},
$$

In $1990-2000$ years the team of scientist from Kuzbass State Technical University made the experiments on controlled destroying of composites and rocks samples with simultaneous measurement of the EMP numbers. In that experiments were investigated phenoplast, textolite, limestone, hornstone, and quartz diorite samples. Plots of dependence between the accumulated numbers of pulses from the mechanical stresses are shown in figures $1-10$.

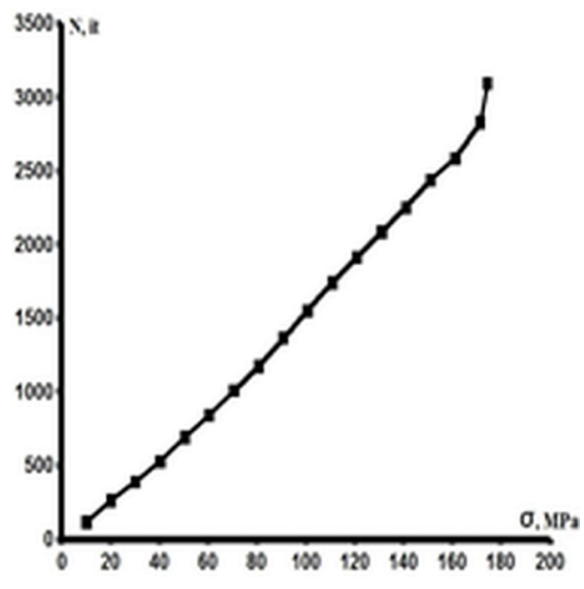

Fig. 1 Sample 1. Phenoplast J13

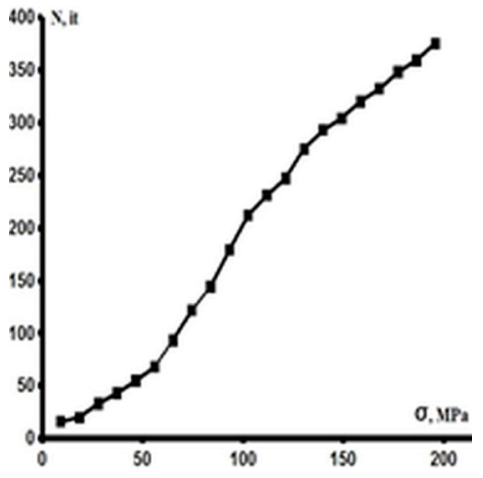

Fig. 2 Sample 2. Phenoplast A39 


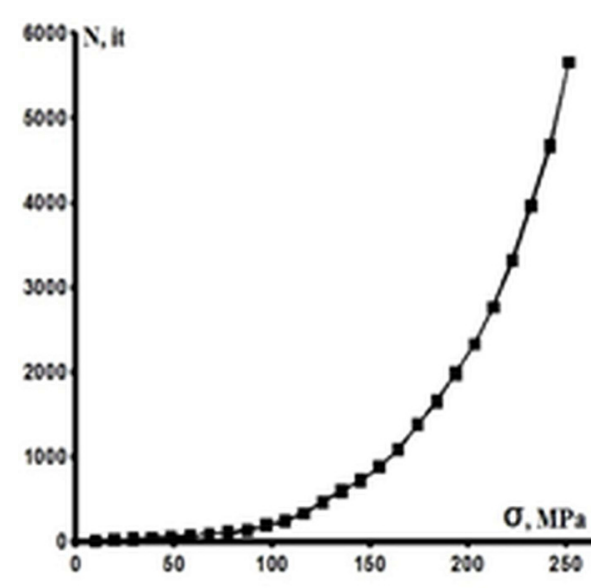

Fig. 3 Sample 3. Textolitet PTM

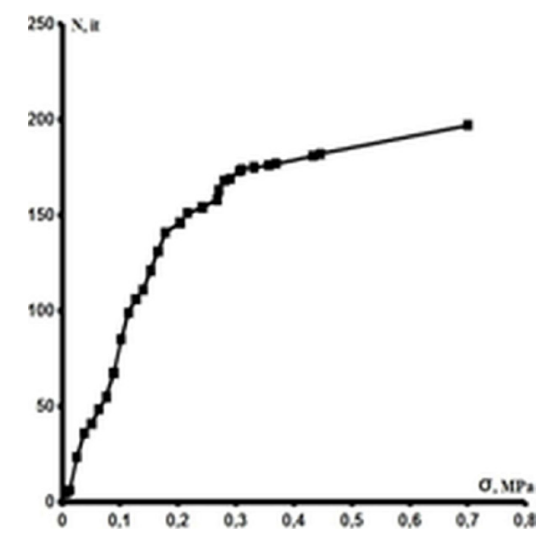

Fig. 5 Sample 5. Limestone 8

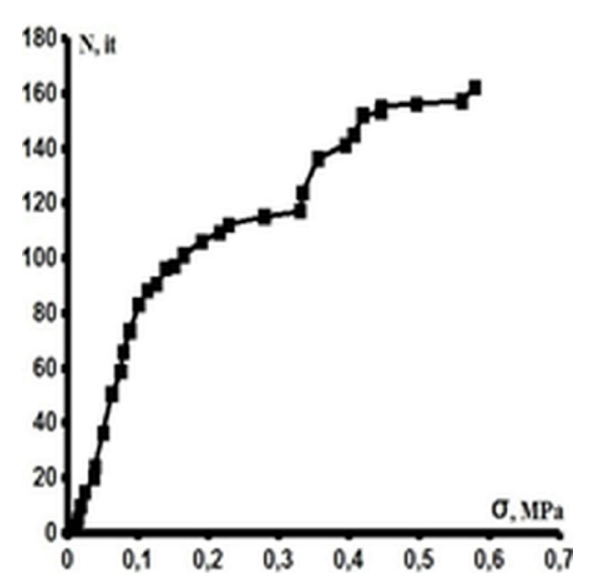

Fig. 7 Sample 7. Quartz diorite 36

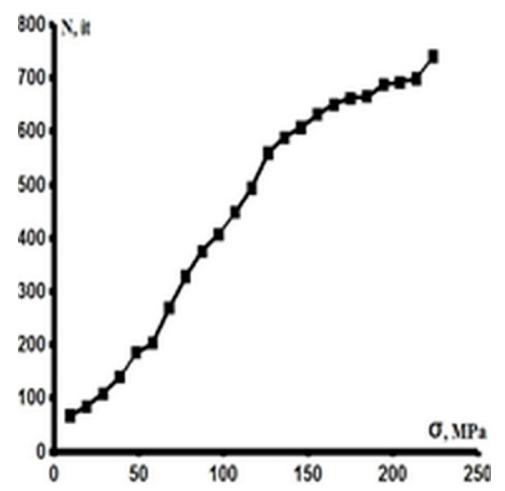

Fig. 4 Sample 4. Textolitet PT

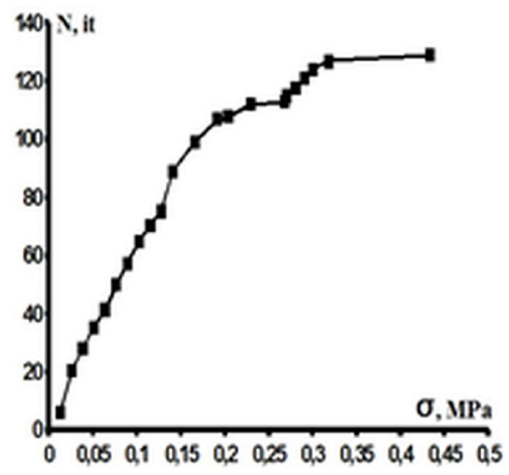

Fig. 6 Sample 6. Limestone 9

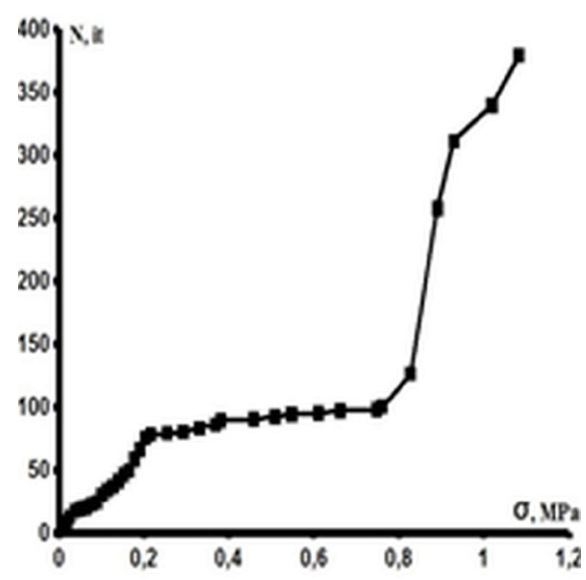

Fig.8 Sample 8. Quartz diorite 37 


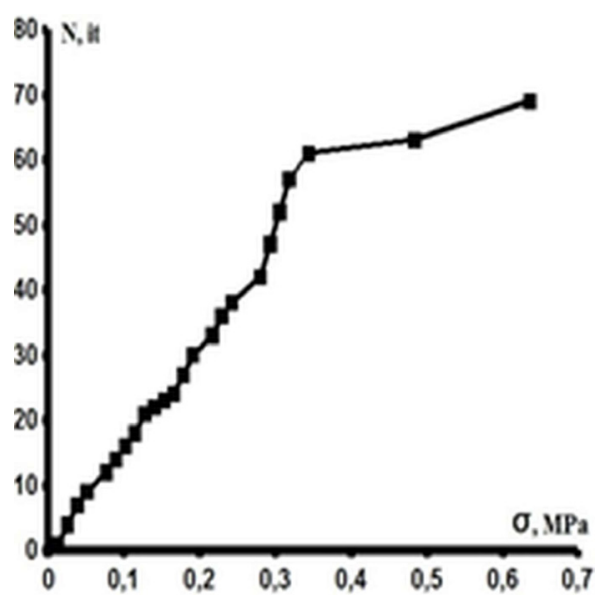

Fig. 9 Sample 9. Hornstone 14

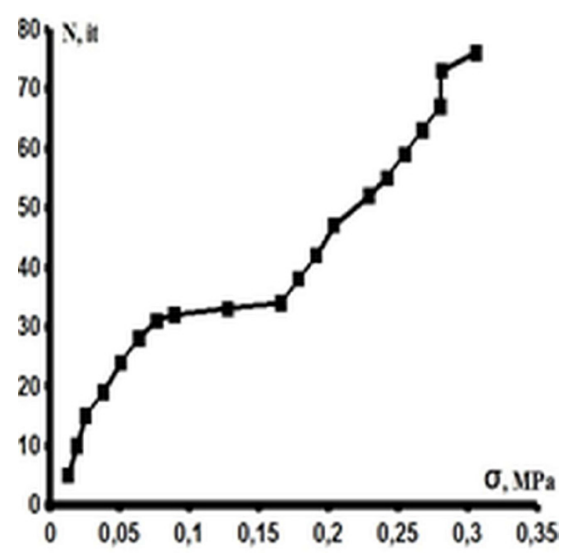

Fig.10 Sample 10. Hornstone 16

Let us test the above formulas $(6,7)$ for these data sets. The unknown parameters $\alpha$ and $\beta$ in dependences $(6,7)$ will be searched using the least squares method as

$$
\sum\left(N_{k}-N_{k}^{T}\right)^{2} \rightarrow \min
$$

the numerical implementation of which using the evolution algorithms is contained in the NLPSolver add-in of the free open-source OO Calc table processor. The accuracy of data fiting we will diagnose by the determination index

$$
R^{2}=1-\frac{\sum\left(N_{k}-N_{k}^{T}\right)^{2}}{\sum\left(N_{k}-N_{a}\right)^{2}},
$$

where $N_{k}$ - experimental data, $N_{k}^{T}$ - theoretical data by (6,7) upper and (11) lower, $N_{a}$ - average of experimental data.

The parameters value $\alpha, \beta$ and the determination index $R^{2}$ were calculated for the given ten samples. The results of the calculations are given in the table 1 .

\begin{tabular}{|c|c|c|c|c|c|}
\hline No & The sample & The formula & $\alpha$ & $\beta$ & $R^{2}$ \\
\hline \multirow{2}{*}{1} & \multirow{2}{*}{ Sample 1} & (6) & 0,003 & 34,956 & 0,997 \\
\hline & & (7) & 0,029 & 35,124 & 0,997 \\
\hline \multirow{2}{*}{2} & \multirow{2}{*}{ Sample 2} & (6) & 0,027 & 39,052 & 0,621 \\
\hline & & (7) & 0,092 & 39,731 & 0,578 \\
\hline \multirow{2}{*}{3} & \multirow{2}{*}{ Sample 3} & (6) & 0,019 & 38,594 & 0,998 \\
\hline & & (7) & 0,181 & 41,986 & 0,998 \\
\hline \multirow{2}{*}{4} & \multirow{2}{*}{ Sample 4} & (6) & $-0,004$ & 34,917 & 0,981 \\
\hline & & (7) & $-0,012$ & 34,982 & 0,971 \\
\hline \multirow{2}{*}{5} & \multirow{2}{*}{ Sample 5} & (6) & $-5,529$ & 34,483 & 0,987 \\
\hline & & (7) & $-0,276$ & 36,764 & 0,915 \\
\hline \multirow{2}{*}{6} & \multirow{2}{*}{ Sample 6} & (6) & $-6,031$ & 33,512 & 0,990 \\
\hline & & (7) & $-2,284$ & 34,611 & 0,952 \\
\hline \multirow{2}{*}{7} & \multirow{2}{*}{ Sample 7} & (6) & $-3,127$ & 35,561 & 0,996 \\
\hline & & (7) & $-0,196$ & 37,725 & 0,976 \\
\hline \multirow[b]{2}{*}{8} & \multirow{2}{*}{ Sample 8} & (6) & 1,826 & 36,993 & 0,856 \\
\hline & & (7) & 1,052 & 36,004 & 0,810 \\
\hline \multirow{2}{*}{9} & \multirow{2}{*}{ Sample 9} & (6) & $-3,131$ & 35,476 & 0,909 \\
\hline & & (7) & $-0,572$ & 35,639 & 0,929 \\
\hline
\end{tabular}

Table 1. The result of the calculations parameters. 


\begin{tabular}{|l|l|l|l|l|l|}
\hline \multirow{2}{*}{10} & \multirow{2}{*}{ Sample 10} & $(6)$ & $-3,131$ & 35,476 & 0,909 \\
\cline { 3 - 6 } & & $(7)$ & $-0,106$ & 36,954 & 0,938 \\
\hline
\end{tabular}

Let us draw some conclusions based on the data in Table 1 .

1) The values of parameter $\beta$, calculated from two different formulas $(6,7)$, coincide in order of magnitude and, moreover, fall into some possible interval of the form $\beta \in[30 ; 40]$.

2) The value of parameter $\alpha$, calculated by formulas $(6,7)$, will be negative for five rock samples and one composite sample. All of these values are physically meaningless.

3) The value of $E$, which in [9] had the meaning of the Young elastic modulus, is a dimensional "fitting" parameter with an unclear physical meaning in this case.

\subsection{New modification of S. N. Zhurkov's formula.}

Let us modify formula (3) as:

$$
\tau=\tau_{0} * \exp (\beta) *\left(\frac{\sigma}{E}\right)^{1-\alpha E},
$$

hence the dependence for the number of pulses (cracks) from the stress is converted to a simpler form

$$
N^{T}(\sigma)=\frac{N_{\max }}{\tau_{0} \cdot \sigma^{\prime}} * \exp (-\beta) * \frac{(\sigma / E)^{\alpha E}}{\alpha} .
$$

Let us calculate the parameters $\alpha, \beta, E$ using the NLPSolver add-on and present its and the kinetic constants of destruction $\Omega$ and $U_{0}$ in Table 2 .

Table 2. The calculated parameters of the dependence (9), the activation volume and the activation energy of failure

\begin{tabular}{|c|c|c|c|c|c|c|c|}
\hline No & The sample & $\boldsymbol{\alpha}$ & $\boldsymbol{\beta}$ & $\boldsymbol{R}^{\mathbf{2}}$ & $\Omega \cdot 10^{27}$ & $U_{0} \cdot 10^{19}$ & $\boldsymbol{E}$ \\
\hline 1 & Sample 1 & 0,192 & 35,121 & 0,997 & 0,781 & 1,422 & 6,107 \\
\hline 2 & Sample 2 & 0,062 & 36,412 & 0,986 & 0,251 & 1,474 & 18,552 \\
\hline 3 & Sample 3 & 0,091 & 39,172 & 0,998 & 0,368 & 1,586 & 42,243 \\
\hline 4 & Sample 4 & 0,127 & 34,834 & 0,971 & 0,515 & 1,410 & 6,432 \\
\hline 5 & Sample 5 & 0,273 & 36,803 & 0,915 & 1,107 & 1,490 & 1,891 \\
\hline 6 & Sample 6 & 0,237 & 35,694 & 0,953 & 0,958 & 1,445 & 2,473 \\
\hline 7 & Sample 7 & 1,131 & 36,133 & 0,967 & 4,581 & 1,463 & 0,519 \\
\hline 8 & Sample 8 & 1,208 & 35,532 & 0,952 & 4,893 & 1,439 & 1,221 \\
\hline 9 & Sample 9 & 0,271 & 36,559 & 0,928 & 1,096 & 1,480 & 1,862 \\
\hline 10 & Sample 10 & 0,309 & 36,882 & 0,938 & 1,255 & 1,493 & 2,246 \\
\hline
\end{tabular}

We can see that

1) the $\alpha$ takes positive value for all samles;

2) the $\beta$ also falls into the same interval $\beta \in[30 ; 40]$;

3) the $R^{2}$ is larger than in table 1 .

\subsection{Some of the possible applications.}

Let us introduce two of the possible applications of the formulas obtained.

The first application is the frost resistance evaluation of the material at a cyclic freezing and calculation the number of full destruction cycles [16]. Let us assume that when the samples are frozen and thawed, the temperature and internal microstresses vary linearly. The number of cycles to full destruction $N_{D}$ of the samples can be calculated from the equa- 
tion $N_{D} \cdot N_{1}=N_{\max }$, where $N_{1}$ - the number of microcracks in first cycle, $N_{\max }$ - the maximum of microcracks before the full destruction. The value $N_{1}$ we can calculate with $(5,11)$ [16] as

$$
N_{1}=\frac{N_{\max }}{\tau_{0}} \int_{0}^{\psi / 2} e^{\frac{-U_{0}}{\kappa}}\left(\frac{\sigma^{\prime} t}{E}\right)^{\frac{\Omega E}{\kappa}-1} d t+\frac{N_{\max }}{\tau_{0}} \int_{\psi / 2}^{\psi} e^{\frac{-U_{0}}{\mu}}\left(\frac{2 \sigma_{A}-\sigma^{\prime} t}{E}\right)^{\frac{\Omega E}{\mu}-1} d t=\frac{N_{\max }}{\tau_{0}} \cdot I,
$$

where $\kappa=k \cdot\left(T_{0}-q t\right) ; \mu=k \cdot\left[T_{0}-80+q t\right] ; T_{0}=293{ }^{0} \mathrm{~K} ; q-$ is the temperature gradient, ${ }^{0} \mathrm{~K} \cdot \mathrm{s}^{-1}$, where $q \cdot \frac{\psi}{2}=40,{ }^{0} \mathrm{~K} ; \sigma_{A}-$ is the amplitude value of microstress in the cycle, $\mathrm{Pa}$, wherein $\sigma^{\prime} \cdot \frac{\psi}{2}=\sigma_{A}$ and $\psi, \mathrm{s}-$ is the period of microstress cycle.

Let us calculate the value $N_{D}$ for sample 3 and sample 8 for example. From the experiment the auxiliary quantities for sample 3 are $\psi=242 \mathrm{~s}, \sigma^{\prime}=1,0378, \mathrm{MPa}^{-1} \mathrm{~s}^{-1}$ consequently, $\sigma_{A}=\sigma^{\prime} \cdot \frac{\psi}{2}=147,3676 \mathrm{MPa}$; the value $q=0,33057{ }^{0} \mathrm{~K} \cdot \mathrm{s}^{-1}$. Similarly for sample 8 $\psi=352 \mathrm{~s}, \sigma^{\prime}=0,00307, \mathrm{MPa} \cdot \mathrm{s}^{-1}$ consequently, $\sigma_{A}=\sigma^{\prime} \cdot \frac{\psi}{2}=0,54032 \mathrm{MPa}$; the value $q=0,22727{ }^{0} \mathrm{~K} \cdot \mathrm{s}^{-1}$. To numerical calculate integrals, we will use 6-points Gauss formula with division of a segment into $\psi$ intervals and implement it in open-source mathematical software Scilab. For sample 3 the value $I=1,105 \cdot 10^{-15}$, and then $N_{D}=\frac{\tau_{0}}{I}=90$; for sample 8 the value $I=1,907 \cdot 10^{-13}$, and then $N_{D}=\frac{\tau_{0}}{I}=17$.

The second application is the calculation of an apparent density of electric currents in geoelecric and geomechanic [17]. They knows that the rock burst source generates mechanical stresses changes and hence quasi-stationary electric field. Let us assume that the source is located in the thickness of rocks deep below the earth's surface. We can calculate 3D current density $j_{3 D} \mathrm{~A} \cdot \mathrm{m}^{-3}$ in it by the formula [17]

$$
j_{3 D}=\frac{2 \pi}{\rho_{1} \varepsilon_{0} \varepsilon_{1}} L Q N^{\prime} \tau_{p},
$$

where $L-$ the linear size of the formed microcracks, $\mathrm{m} ; N^{\prime}$ is calculated by the $(5) ; \tau_{p}-$ the time of relaxation of charges at the top of the crack, (s.); $Q$ - the linear charge density per unit length of the crack front, $\mathrm{C} / \mathrm{m} ; \varepsilon_{0} \varepsilon_{1}$ - the absolute dielectric permeability of rocks in layer $1, F / m ; \rho_{1}$ - specific electrical resistivity of rocks contained the source, Ohmm.

The number of microcracks accumulated by the time of the rock burst in the volume unit can be determined from (1) $n \approx 0,037 L^{-3}$. Let us take a typical values of this parameters (for samples 7 and 8) [17]: $L=2 \cdot 10^{-4} \mathrm{~m}, Q=5 \cdot 10^{-11} \mathrm{C} / \mathrm{m}, \tau_{p}=10^{-5} \mathrm{~s}$, $\varepsilon_{0} \varepsilon_{1} \approx 8,854 \cdot 10^{-12} \mathrm{~F} / \mathrm{m}, \quad \rho_{1}=10^{3}$ Ohmm, hence $j_{3 D}=\frac{328,73}{\tau}$. From (8) with $\sigma_{p} \approx 0,0433 \mathrm{MPa}$ the value $\tau=1318,71 \mathrm{~s}$ and hence $j_{3 D}=0,249 \mathrm{~A} \cdot \mathrm{m}^{-3}$.

\section{Conclusion}


In this article, a we proposed a new modification of Zhurkov's formula. The investigation showed that: 1) it is suitable for calculating the durability of both the sample compression and its tensile; 2) it is suitable for rock and composites materials.

The disadvantage of the proposed formula are: 1) physical meaningless by the additional constant E; 2) the formula (2) contain three unknown parameters which makes it harder to find them.

\section{References}

1. S. N. Zhurkov, Int. J. Fract. Mech, 1(4), 311 (1964)

2. K. I. DeVries, D. K. Roylance, M. I. Williams, Int. J. Fract., 7197 (1971)

3. V. R. Regel, A. L. Slutsker, and E. E. Tomashevskii, The Kinetic Nature of the Solid Strength (Science, Moscow, 1974)

4. D. Roylace, Int. J. Fract. 21, 107 (1983)

5. J. Smook, W. Hamersma, A. Pennings, J. Mater. Sci., 19, 1359 (1984)

6. D. J. Dijkstral, J. C. M. Torfs, and A. J. Pennings, Colloid Polym Sci 267, 866, (1989)

7. R. Salganik, V.A. Gotlib, Int. J. Fract. 101, 181 (2000)

8. A. I. Slutsker, Phys. Solid State, 46(9), 1658 (2004).

9. V. I Vettegen, V. B. Kulik, S.V. Bronnikov, Tech. Phys. Lett., 31(11), 969 (2005)

10. S. O. Lazarev, Yu. K. Mikhailov, Phys. Solid State, 47(5), 984 (2005)

11. V. S. Kuksenko, Phys. Solid State, 47(5), 812 (2005)

12. L. Mishnaevsky, P. Brøndsted, Int. J. Fract. 144, 149 (2007)

13. V. V. Shevelev, Glass Phys. and Chem., 35(6), 567 (2009)

14. V. P. Efimov, V. S. Nikiforovsky, J. Mining Sci., 46(3), 260 (2010)

15. E. Suhir, J. Mater Sci: Mater Electron, 28, 11689 (2017)

16. O.P. Afinogenov, V.V. Ivanov, Theoretical bases ensure durability non-rigid garments of roads mining (KuzSTU, Kemerovo, 2001)

17. V. V. Ivanov, Physical bases of electromagnetic processes at formation of the hearth of destruction in an array of rocks (KuzSTU, Kemerovo, 1994) 